\title{
Complete Hexose Isomer Identification with Mass Spectrometry
}

\author{
Gabe Nagy, Nicola L. B. Pohl
}

Department of Chemistry, Indiana University, Bloomington, IN 47405, USA

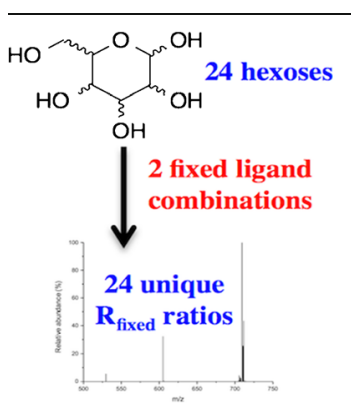

provides a key step towards the goal of complete de novo carbohydrate analysis.

Keywords: Monosaccharide, Hexose isomers, Sugar sequencing, Mass spectrometry, Kinetic resolution, Carbohydrates, Carbohydrate analysis, Chirality, Configuration determination

\begin{abstract}
The first analytical method is presented for the identification and absolute configuration determination of all 24 aldohexose and 2-ketohexose isomers, including the $D$ and $L$ enantiomers for allose, altrose, galactose, glucose, gulose, idose, mannose, talose, fructose, psicose, sorbose, and tagatose. Two unique fixed ligand kinetic method combinations were discovered to create significant enough energetic differences to achieve chiral discrimination among all 24 hexoses. Each of these 24 hexoses yields unique ratios of a specific pair of fragment ions that allows for simultaneous determination of identification and absolute configuration. This mass spectrometric-based methodology can be readily employed for accurate identification of any isolated monosaccharide from an unknown biological source. This work
\end{abstract}

Received: 24 September 2014/Revised: 19 December 2014/Accepted: 19 December 2014/Published Online: 5 February 2015

\section{Introduction}

$\mathrm{C}$ arbohydrates serve key structural and functional roles in nature that include a wide range of biological processes such as cell-cell recognition, protein folding and degradation, immune/host-pathogen responses, and metabolism [1-5]. However, carbohydrate sequencing has significantly lagged capabilities in sequencing nucleic acids and proteins. Compared with these other biomolecules, polymers and oligomers of carbohydrates are more structurally complex because of their large range of stereochemical differences, absolute configuration $(D$ or $L$ ), anomeric configurations ( $\alpha$ or $\beta$ ), and linkage positions $[2,4,6,7]$. This high degree of structural complexity has hindered accurate structural analyses of glycans, so much so that a United States National Academy of Sciences report issued in 2012 has called for the development of technology over the next 10 years to purify, identify, and determine the structures of all the important glycoproteins, glycolipids, and polysaccharides in any biological sample [8].

Currently, structural elucidation projects usually begin with hydrolysis of the glycan into its constituent monosaccharides

Electronic supplementary material The online version of this article (doi:10.1007/s13361-014-1072-z) contains supplementary material, which is available to authorized users.

Correspondence to: Nicola Pohl; e-mail: npohl@indiana.edu for their accurate identification, often by a gas chromatographymass spectrometry (GC-MS) method [9]. Unfortunately, this method is limited by the need for sample derivatization as well as convoluted chromatograms from the resulting mixture of $\alpha / \beta$ and furanose/pyranose forms $[10,11]$. Presently, much attention is given to the structural elucidation of mammalian $\mathrm{N}$ glycans and glycosaminoglycans [12]. This task is relatively simpler than de novo carbohydrate sequencing because only a small subset of monosaccharide subunits are involved, and all these subunits are believed to be known. However, the presumption of such a limited set of monosaccharide building blocks does not hold up when faced with an unknown biological sample from the many diverse branches of life. The entire set of possible monosaccharide isomers must be taken into account in its analysis. For accurate identification of such unknown glycan structures, reliable high-throughput methods for complete monosaccharide analysis are needed first $[2-4,6$, 13]. Monosaccharide constituents are often identified by mass alone into classes such as hexose or amino-hexose as a means of preliminary structural analysis. However, it is unclear whether a single technique such as mass spectrometry could be used to further distinguish between all the possible hexoses, for example. Neutral hexoses (Figure 1) can be described as 12 diastereomers, each composed of two, $D$ and $L$, mirror-image enantiomers, for a total of 24 different isomers. Furthermore, these can be divided into the 16 aldohexose isomers (allose, altrose, galactose, glucose, gulose, idose, mannose, and talose), 

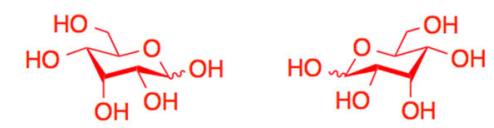

D-allose (D-All)

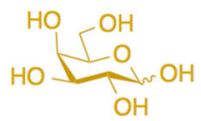

D-galactose (D-Gal)

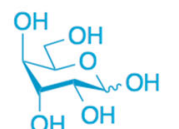

D-gulose (D-Gul)

$$
\mathrm{HO}-\mathrm{OH}
$$

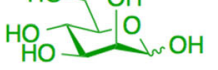

D-mannose (D-Man)
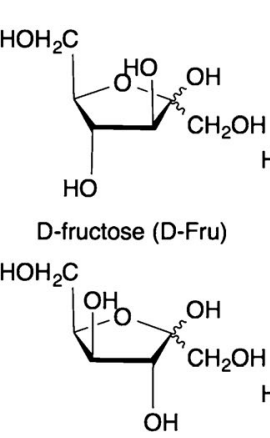

Linu

L-allose (L-All)

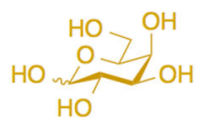

L-galactose (L-Gal)

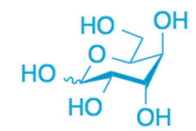

L-gulose (L-Gul)

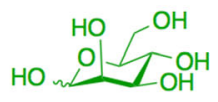

L-mannose (L-Man)

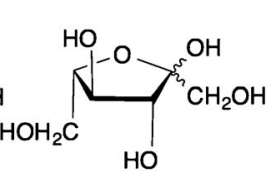

L-fructose (L-Fru)<smiles>O=C(O)C1C(O)C(O)C(O)C1O</smiles>

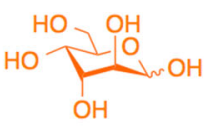

D-altrose (D-Alt)

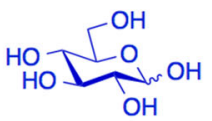

D-glucose (D-Glc)

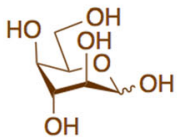

D-idose (D-Ido)

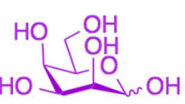

D-talose (D-Tal)

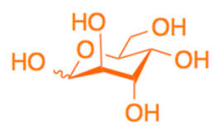

L-altrose (L-Alt)<smiles>OCC1(O)C(O)C(O)C(O)C(O)C1O</smiles>

L-glucose (L-Glc)

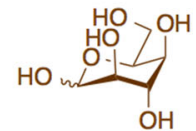

L-idose (L-Ido)

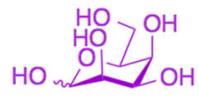

L-talose (L-Tal)

D-sorbose (D-Sor)

$\mathrm{HOH}_{2} \mathrm{C}$<smiles>CC1OC(O)(CO)C(O)C1O</smiles><smiles>OCC(O)C(CO)C(O)(O)CO</smiles>

D-psicose (D-Psi)

L-psicose (L-Psi)

$\mathrm{HOH}_{2} \mathrm{C}$

L-sorbose (L-Sor)

Figure 1. The 24 hexose monosaccharide isomers

and the 8 ketohexose isomers (fructose, psicose, sorbose, and tagatose). Obviously, the major question arises as to whether or not simultaneous discrimination and identification of all 24 neutral monosaccharide isomers is even possible. Their only structural differences (as shown in Figure 1) are the axial or equatorial hydroxyl group permutations at $\mathrm{C} 2$, $\mathrm{C} 3$, and $\mathrm{C} 4$ for the aldohexoses, and $\mathrm{C} 3$ and $\mathrm{C} 4$ for the ketohexoses. From this structural similarity, it is evident that minimal energetic differences will exist between all 24 isomers. Without sufficient energetic differences between them, discrimination and separation would be impossible. The central challenge then comes down to finding a set of ligands that bind to each of the 24 isomers to create large enough energetic differences to accurately discriminate amongst the isomers within error bars-a daunting task given the incredible difficulty seen in designing artificial receptors that can discriminate amongst sugar stereoisomers. These sugar receptors have had only limited success in achieving stereoselectivity based on the small binding energy differences between stereoisomers [14, 15].

Other analytical techniques employed for monosaccharide analysis include high-performance anion-exchange chromatography (HPAEC), HPLC, and NMR [16-23]. Some disadvantages associated with each include the absence of measurable J values and overlap of proton signals in NMR, and long column regeneration time between runs and inability to determine absolute configuration in HPAEC [16-23]. Although HPLC provides one of the more popular methods for monosaccharide analysis, it has certain limitations [24-30]. Often times, sample preparation or derivatization is needed and, even in the case of chiral columns, there has not been as large or as complete a set of monosaccharides discriminated from one another as presented here. Furthermore, identification of an unknown monosaccharide is limited by overlapping retention times from the $\alpha / \beta$ and furanose/pyranose forms. The possibility was explored that mass spectrometric methods could overcome these problems and also discriminate monosaccharide identity and absolute configuration. Previous studies have not demonstrated simultaneous discrimination of diastereomers and enantiomers.

Mass spectrometry is often thought of as a chirally blind technique - one that is unable to discriminate between enantiomers, let alone be used for complete analysis of a large isomeric set. However, mass spectrometry has certain inherent advantages compared with the aforementioned analytical techniques previously employed for monosaccharide analysis $[1,17$, 
$18,22,31,32]$. Specifically, mass spectrometry does not require any solvent or stationary phase, and also provides fast, accurate, and sensitive sample analysis. To date, monosaccharide analysis techniques in mass spectrometry result in similar $\mathrm{MS}^{1}$ spectra for diastereomers and similar $\mathrm{MS}^{2}$ spectra for enantiomers. In other words, no previous mass spectrometric methods have been developed to simultaneously discriminate diastereomers and enantiomers of a complete set of isomers [33-36].

\section{Experimental}

\section{Conventional Kinetic Method}

Given earlier findings with carbohydrate-active enzymes discriminating amongst different glycan substrates through kinetic rather than purely binding affinity differences [37, 38], it was set out to discover much smaller carbohydrate-binding ligands that could discriminate amongst all the neutral hexoses through kinetic and not just thermodynamic differentiation steps. A related thought process forms the basis of Cooks' kinetic method, perhaps the most widely applied chiral mass spectrometric technique [16-18, 39-45]. This method measures the dissociation rates of cluster ions composed of a metal, chiral reference, and analyte of interest. Fragmentation patterns are affected by the strength of the metalbound analyte ligand, which varies with chirality of the analyte. Specifically, the basis of the kinetic method is the formation of a singly charged, trimeric ion complex via electrospray ionization (ESI), where $\mathrm{M}^{\mathrm{II}}$ is the divalent metal cation, reference is the chiral reference, $\mathrm{A}$ is the monosaccharide analyte, and $k_{1}$ and $k_{2}$ are the two competitive dissociation pathways (Figure 2). It can be seen that the two diastereomeric fragment ions are either from the neutral loss of an analyte or neutral loss of a reference molecule, both from the initial trimeric ion complex (Figure 2). A branching ratio term, $\mathrm{R}$ value, can quantify a relationship between the two diastereomeric fragment ions as a measure of their relative intensities (Equation 1). When an individual enantiomer is present, the $R$ value can become either $R_{D}$ or $R_{L}$, and the chiral discrimination between an enantiomeric pair

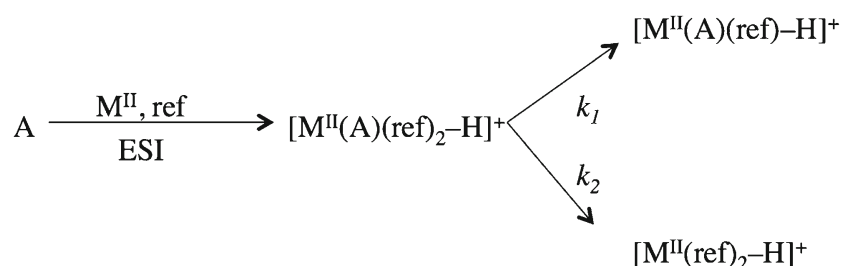

Figure 2. Formation of divalent metal-bound trimeric ion complex via electrospray ionization, and subsequent fragmentation through collision-induced dissociation in conventional kinetic method can be represented by the ratio between $R_{D}$ and $R_{L}$ in the form of the $R_{\text {chiral }}$ term where a value further from unity is desired (Equation 2).

$$
R=\frac{\left[M^{I I}(A)(\text { ref })-H\right]^{+}}{\left[M^{I I}(\text { ref })_{2}-H\right]^{+}}
$$

$$
R_{\text {chiral }}=\frac{R_{D}}{R_{L}}=\frac{\left[M^{I I}\left(A_{D}\right)(\text { ref })-H\right]^{+} /\left[M^{I I}(\text { ref })_{2}-H\right]^{+}}{\left[M^{I I}\left(A_{L}\right)(\text { ref })-H\right]^{+} /\left[M^{I I}(\text { ref })_{2}-H\right]^{+}}
$$

\section{Fixed Ligand Kinetic Method}

Although this conventional kinetic method has been widely applied, it is limited by the lack of chiral selectivity in certain cases, as well as isomeric complexes formed in the gas phase from the multiple possible deprotonation $(-\mathrm{H})$ sites. One modification to help alleviate these problems and, furthermore, increase chiral selectivity, is the fixed ligand kinetic method, also developed by Cooks and co-workers [17, 18, 41, 46-48]. Here, the initial trimeric ion complex formed is $\left[\mathrm{M}^{\mathrm{II}}(\mathrm{A})(\mathrm{ref})(\mathrm{FL}-\mathrm{H})\right]^{+}$, while the two diastereomeric fragment ions from the two competitive dissociation pathways are $\left[\mathrm{M}^{\mathrm{II}}(\mathrm{A})(\mathrm{FL}-\mathrm{H})\right]^{+}$and $\left[\mathrm{M}^{\mathrm{II}}(\mathrm{ref})(\mathrm{FL}-\right.$ $\mathrm{H})]^{+}$, where $\mathrm{FL}$ is the fixed ligand (Figure 3). It can be seen that an easily deprotonated fixed ligand molecule will replace one of the chiral references in the original trimeric ion complex (Figure 3). More importantly, the fixed ligand is not lost in CID and thus the deprotonation site is confined to it, which avoids the formation of various isomeric structures. Chiral discrimination is improved because more chiral interactions occur in the diastereomeric fragments compared with the conventional kinetic method. Once again, a branching ratio, $\mathrm{R}_{\text {fixed, }}$, relates the two fragment ions to one another as a measure of their relative intensities (Equation 3). When one specific enantiomer is present, $R_{\text {fixed }}$ can become either $\mathrm{R}_{\mathrm{D} \text {-fixed }}$ or $\mathrm{R}_{\mathrm{L} \text {-fixed }}$ (Equations 4 and 5). Chiral discrimination between an enantiomeric pair can be quantified

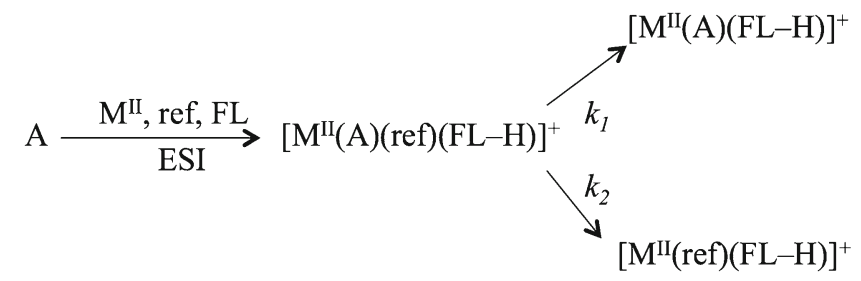

Figure 3. Formation of divalent metal-bound trimeric ion complex via electrospray ionization, and subsequent fragmentation through collision-induced dissociation in fixed ligand kinetic method 
(Equation 6) through the $\mathrm{R}_{\text {chiral-fixed }}$ term, where a value further from unity is desired.

$R_{\text {fixed }}=\frac{\left[M^{I I}(A)(F L-H)\right]^{+}}{\left[M^{I I}(r e f)(F L-H)\right]^{+}}$

$R_{D-\text { fixed }}=\frac{\left[M^{I I}\left(A_{D}\right)(F L-H)\right]^{+}}{\left[M^{I I}(\text { ref })(F L-H)\right]^{+}}$

$R_{L-f i x e d}=\frac{\left[M^{I I}\left(A_{L}\right)(F L-H)\right]^{+}}{\left[M^{I I}(r e f)(F L-H)\right]^{+}}$

$R_{\text {chiral-fixed }}=\frac{R_{D-\text { fixed }}}{R_{L-\text { fixed }}}$

Chiral separation in the fixed ligand kinetic method comes from Equation 7, where $\mathrm{R}_{\text {fixed }}$ is the fixed ligand branching ratio, $\mathbf{R}$ is the gas constant, $\Delta(\Delta G)$ is the change in Gibbs' free energy, and $\mathrm{T}_{\text {eff }}$ is the average temperature of the activated complexes $[17,18,41,46-$ 48]. Small differences in free energy between the diastereomeric fragment ion complexes will result in larger differences in their branching ratio, $\mathrm{R}_{\text {fixed }}$, values. More specifically, differences in the stability (and thus energetics) of the diastereomeric fragment ion complexes will result in different relative intensities and, thus, unique $\mathrm{R}_{\text {fixed }}$ values for each neutral hexose enantiomer. Figure 4 illustrates a free energy diagram representation of the fixed ligand kinetic method.

$\ln \left(R_{\text {chiral-fixed }}\right)=\frac{\Delta(\Delta G)}{\boldsymbol{R} T_{\text {eff }}}$

\section{Mass Spectrometric Methodology}

Previous monosaccharide work with the Cooks' kinetic method has focused on the $\mathrm{R}_{\text {chiral-fixed }}$ term for enantiomeric pairs of the "common" sugars (galactose, glucose, mannose, and fructose) $[20,41,46,49]$. However, none of these studies have incorporated any of the "rare" sugars (allose, altrose, gulose, idose, talose, psicose, sorbose, and tagatose) into their results. Furthermore, these previous studies have not shown simultaneous discrimination of diastereomers and enantiomers, nor have they included all 24 neutral hexose isomers. It was unclear whether any set of ligands would be able to differentiate amongst so many closely related structures. The challenge was to develop a method that would allow all 24 neutral hexose isomers to be discriminated from one another, with simultaneous absolute configuration determination.

Whereas the Cooks' kinetic method focuses solely on the $\mathrm{R}_{\text {chiral-fixed }}$ term to relate one individual pair of $D / L$ enantiomers, a novel $\mathrm{R}_{\text {fixed }}$ range term has been developed that allows the simultaneous relation of multiple sets of $D / L$ enantiomers to one another and determine absolute configuration. From Equations 4 and 5, it can be seen that each monosaccharide will provide a fixed ligand branching ratio, either $\mathrm{R}_{\mathrm{D} \text {-fixed }}$ or $\mathrm{R}_{\mathrm{L} \text {-fixed }}$, based on the enantiomer (e.g., $\mathrm{R}_{\mathrm{D}-\mathrm{All}}, \mathrm{R}_{\mathrm{L} \text {-All }}, \mathrm{R}_{\mathrm{D} \text {-Alt, }}$ $\mathrm{R}_{\mathrm{L}-\mathrm{Alt}}, \mathrm{R}_{\mathrm{D} \text {-Gal, }}, \mathrm{R}_{\mathrm{L} \text {-Gal }}$, etc...). Simultaneous monosaccharide identification and absolute configuration determination can be found if each of the 24 hexose isomers has a unique and nonoverlapping $\mathrm{R}_{\text {fixed }}$ range; in other words if $\mathrm{R}_{\mathrm{D}-\mathrm{All}}, \mathrm{R}_{\mathrm{L}-\mathrm{All}}, \mathrm{R}_{\mathrm{D}-\mathrm{Alt}}$, $\mathrm{R}_{\mathrm{L}-\mathrm{Alt}}, \mathrm{R}_{\mathrm{D}-\mathrm{Gal}}, \mathrm{R}_{\mathrm{L}-\mathrm{Gal}}$, etc... do not overlap with one another in their $\mathrm{R}_{\text {fixed }}$ ranges, these 24 hexose isomers are discriminated from one another.

\section{Materials}

All neutral monosaccharides, chiral references, fixed ligands, and metal salts were purchased from Carbosynth (Berkshire, UK) and Sigma Aldrich (Milwaukee, WI, USA) without any further purification ( $\geq 95 \%)$ : monosaccharides $(D / L$-allose, $D /$ $L$-altrose, $D / L$-galactose, $D / L$-glucose, $D / L$-gulose, $D / L$-idose, $D / L$-mannose, $D / L$-talose, $D / L$-fructose, $D / L$-psicose, $D / L$-sorbose, and $D / L$-tagatose $)$, metal salts $\left(\mathrm{CuCl}_{2}, \mathrm{NiCl}_{2}, \mathrm{MnCl}_{2}\right.$, and

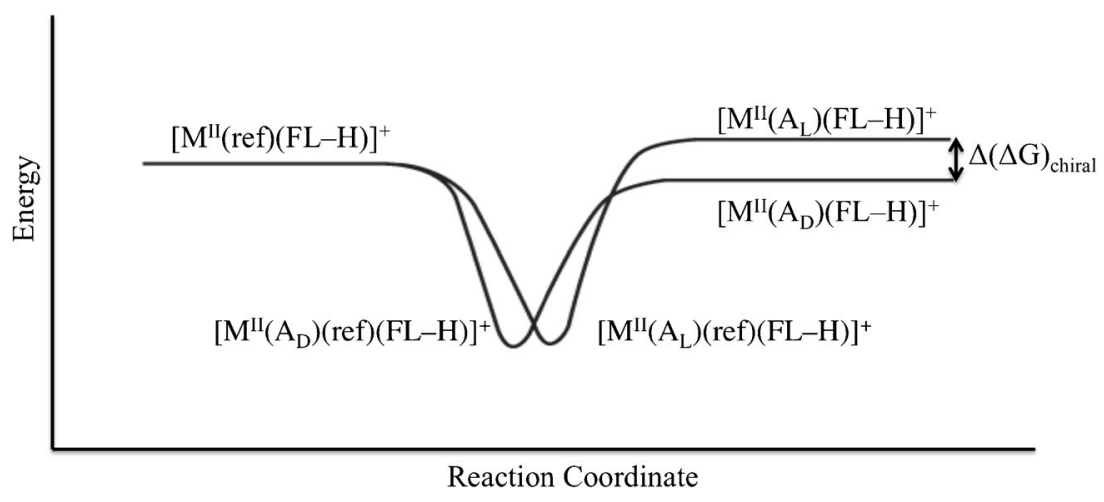

Figure 4. Free energy diagram of fixed ligand kinetic method that illustrates energetic differences of diastereomeric fragment ion complexes 
$\mathrm{Na}_{2} \mathrm{~B}_{4} \mathrm{O}_{7}-10 \mathrm{H}_{2} \mathrm{O}$ ), chiral references ( $L$-Serine and $L$-Aspartic acid), and fixed ligand molecules ( $L$-Phe-Gly, $5^{\prime} \mathrm{CMP}$, and $5^{\prime}$ GMP- $\mathrm{Na}_{2}$ ). All stock solutions were prepared with HPLC grade water and HPLC grade methanol in 50:50 (v/v) mixtures. For the conventional kinetic method, the relative concentrations used were 1:4:4, metal:chiral reference:analyte, whereas, with the fixed ligand method, the relative concentrations were 1:4:4:4, metal:chiral reference:fixed ligand:analyte. The final concentrations used in the conventional kinetic method were 5 $\times 10^{-5} \mathrm{M}$ divalent metal cation, $2 \times 10^{-4} \mathrm{M}$ chiral reference, and $2 \times 10^{-4} \mathrm{M}$ analyte. In the fixed ligand method, the final concentrations used were $5 \times 10^{-5} \mathrm{M}$ divalent metal cation, $2 \times$ $10^{-4} \mathrm{M}$ chiral reference, $2 \times 10^{-4} \mathrm{M}$ fixed ligand, and $2 \times 10^{-4}$ $\mathrm{M}$ analyte. It is important to note that traces of formic acid were added to the $5^{\prime} \mathrm{GMPNa}_{2}$ stock solution and allowed to sit at room temperature for $2 \mathrm{wk}$ prior to the experiment [46].

\section{Mass Spectrometry Conditions}

All experiments were performed on the ion trap portion of an LTQ Orbitrap XL from Thermo Scientific (San Jose, CA, USA), with an ESI source. The Xcalibur software was used for data acquisition. The source conditions used were: $5 \mathrm{kV}$ spray voltage, $0 \mathrm{~V}$ capillary voltage, $150^{\circ} \mathrm{C}$ capillary temperature, $40 \mathrm{~V}$ tube lens voltage, and 6 units sheath gas flow rate. The flow rate used was $5 \mu \mathrm{L} / \mathrm{min}$ with a maximum ion injection time of $500 \mathrm{~ms}$. In $\mathrm{MS}^{2}$, the parent ion was isolated through the resonance ejection of all other ions, except the $\mathrm{m} / \mathrm{z}$ of interest. Resonance excitation was applied on these selected ions to cause collision-induced dissociation (CID). $\mathrm{MS}^{2}$ fragmentation and CID conditions were: $10 \mathrm{~m} / \mathrm{z}$ isolation width, $5 \%-20 \%$ normalized collision energy ( $\% \mathrm{NCE}), 30 \mathrm{~ms}$ activation time with wideband activation. Collision energy in terms of $\mathrm{mV}$ applied for collision-induced dissociation was approximately 5-50 mV, and was optimized for each kinetic method combination and kept constant for all of the 24 hexose isomers. Each spectrum represents an average of 32 scans with three microscans included. All results represent triplicate trials with standard deviations included.

\section{Results and Discussion}

The goal was to first optimize a set of ligands for the identification and absolute configuration determination of the 16 aldohexose isomers. Once these specific conditions were found, they were then applied to the eight ketohexose isomers. It was decidedly easier to first focus on the larger group of isomers (aldohexoses) and then use these optimized conditions on the smaller group of isomers (ketohexoses) to illustrate the discrimination and absolute configuration determination of all 24 neutral hexose monosaccharide isomers.

\section{Aldohexoses-Conventional Kinetic Method}

First, the ability of the conventional kinetic method to resolve all 16 aldohexose isomers was tested. Chiral references and divalent metal cations were initially chosen based on their known ability from previous studies to form the trimeric ion complexes in high abundances, as well as undergo competitive dissociation to form both diastereomeric fragment ions [20,40, $41,46,47,49]$. Furthermore, transition metals contain $d$-electronic orbitals that increase chiral recognition as well as being oxophilic, which is preferred for monosaccharide analytes. Chiral references should have similar size and functional groups $(\mathrm{OH})$ as the analyte of interest $[20,40,41,46,47$, 49]. Although boron readily forms cis-diols with the hydroxyl groups of monosaccharides [38, 50, 51], $\mathrm{B}^{\mathrm{III}}$ proved useless as the central metal cation to form the desired trimeric ion complex. Two different conventional kinetic method combinations of metal cations and chiral references, $\mathrm{Mn}^{\mathrm{II}} / L$-Asp and $\mathrm{Cu}^{\mathrm{II}} / L$ Ser, were employed for all 16 monosaccharides. It was observed that $\mathrm{R}$ values were both too similar from isomer to isomer and irreproducible (i.e., large error bars), caused by the lack of significant energetic differences in the diastereomeric fragment ion complexes formed for each of the 16 different aldohexose isomers with each conventional kinetic method combination applied (see Supporting Information). From these results, no conventional kinetic method combinations were pursued. It is clear that these two combinations cannot resolve the 16 aldohexose isomers, let alone the goal to combine them with the eight ketohexose isomers, for all 24 neutral hexoses. Clearly, a system with the minimum number of steps (combinations) is desired for the development of a robust method amenable to high throughput carbohydrate analysis.

\section{Aldohexoses-Fixed Ligand Kinetic Method}

Since the conventional kinetic method was insufficient, the fixed ligand approach was applied. The fixed ligands chosen were $L$-Phe - Gly, $5^{\prime} \mathrm{CMP}$, and $5^{\prime} \mathrm{GMP}$, based on previous literature [41, 46-48]. Fixed ligands must contain some inherent chirality and, preferably, an aromatic group that could increase chiral discrimination from cation- $\pi$ interactions with the central, divalent metal cation [32, 46, 47]. More specifically, the exact combinations were: $\mathrm{Mn}^{\mathrm{II}} / L$-Asp/L-Phe - Gly, $\mathrm{Ni}{ }^{\mathrm{II}} / L$-Asp $/ 5^{\prime} \mathrm{CMP}, \mathrm{Ni}^{\mathrm{II}} / L$-Asp $/ 5^{\prime} \mathrm{GMP}$, and $\mathrm{Cu}^{\mathrm{II}} / L-\mathrm{Ser} / 5^{\prime}$ GMP. It was determined that if $\mathrm{Mn}^{\mathrm{II}} / L$-Asp/ $L$-Phe - Gly, and $\mathrm{Cu}^{\mathrm{II}} / L$-Ser/5'GMP are applied in two different steps to all 16 aldohexose isomers, monosaccharide identification and absolute configuration determination is achieved. Work with the other fixed ligand combinations produced insufficient aldohexose isomer identification (see Supporting Information). A few $\mathrm{MS}^{2}$ plots of the fragmentation of the parent ion, $\left[\mathrm{Cu}^{\mathrm{II}}(\mathrm{A})(L-\right.$ Ser) $\left.\left(5^{\prime} \mathrm{GMP}-\mathrm{H}\right)\right]^{+}$are shown (Figure 5) to illustrate how $\mathrm{R}_{\text {fixed }}$ is found, as well as to provide an example of how fragment ion abundances differ from monosaccharide to monosaccharide (see the Supporting Information for $\mathrm{MS}^{1}$ spectra).

\section{Complete Aldo/Keto-Hexose Discrimination}

Since a set of fixed ligand combinations, $\mathrm{Mn}^{\mathrm{II}} / L$-Asp $/ L$-Phe Gly and $\mathrm{Cu}^{\mathrm{II}} / L$-Ser $/ 5^{\prime} \mathrm{GMP}$, that could resolve the 16 

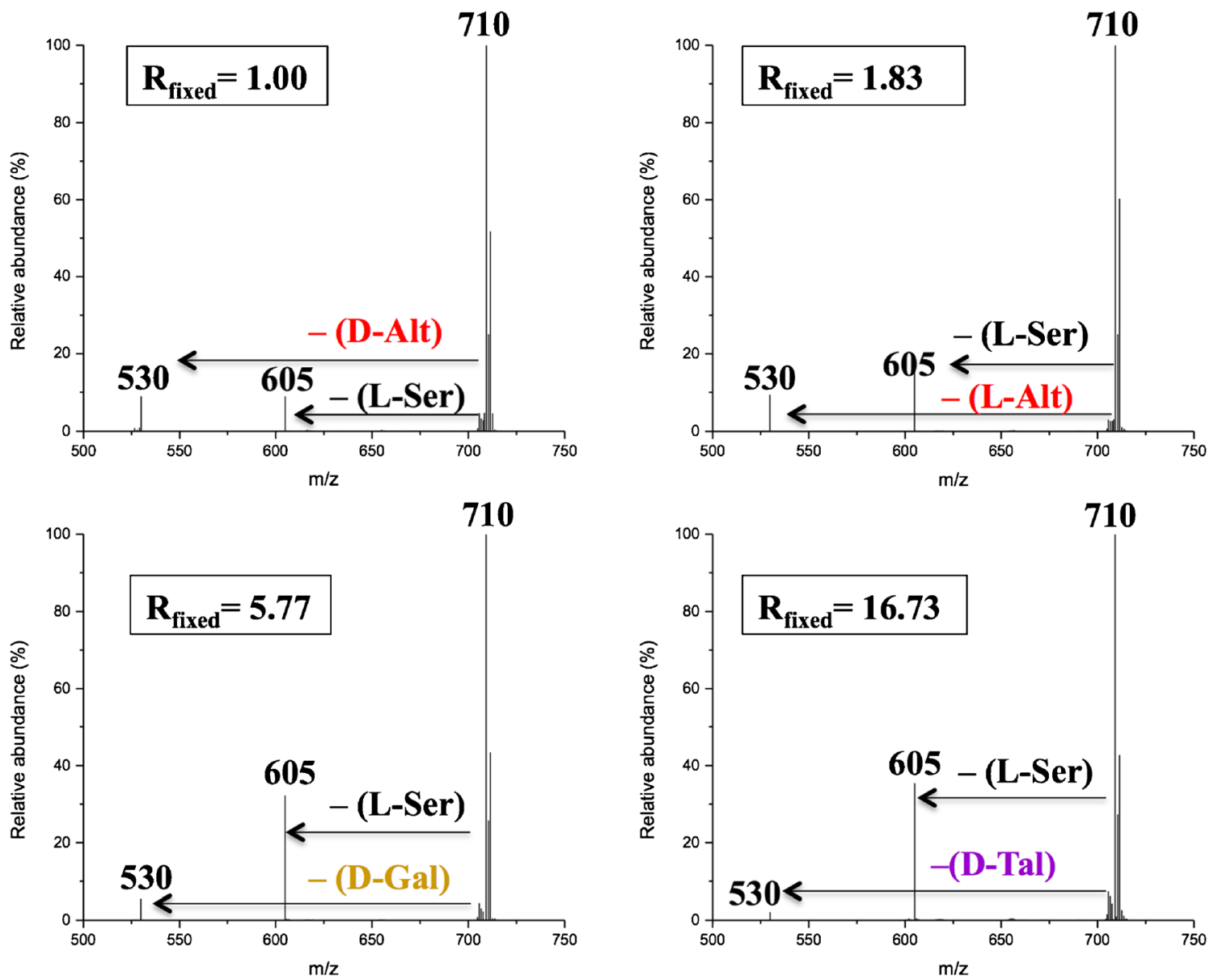

Figure 5. Fixed ligand kinetic method $\mathrm{MS}^{2}$ spectra with Cu", $L$-Ser, and $5^{\prime}$ GMP, for $D$-Alt, $L$-Alt, $D$-Gal, and $D$-Tal at $10 \%$ NCE and 30 ms activation time

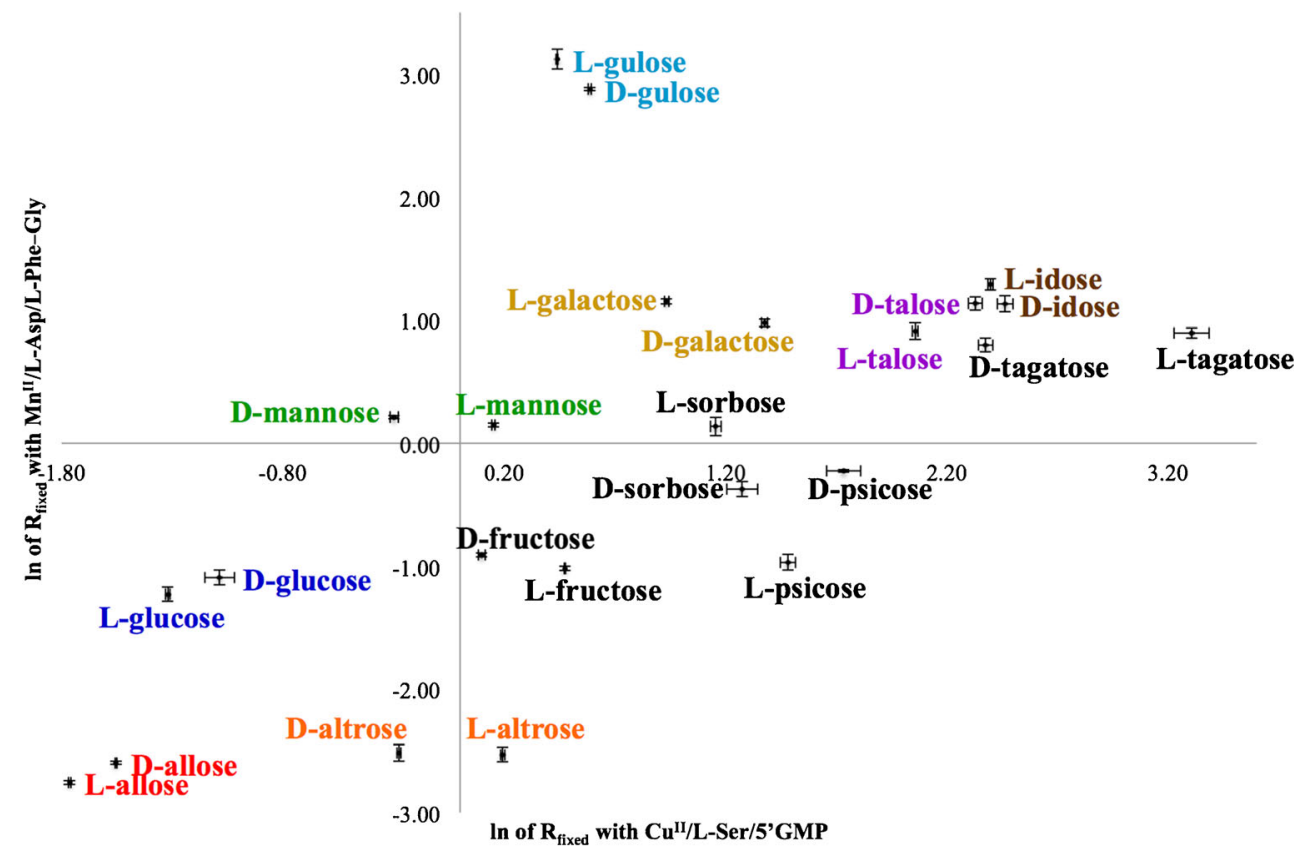

Figure 6. 2-D natural log-natural log plot of $\mathrm{R}_{\mathrm{fixed}}$ with $\mathrm{Cu}^{\prime \prime} / L$-Ser/5'GMP on the x-axis, and $\mathrm{R}_{\mathrm{fixed}}$ with $M \mathrm{Mn}^{\prime \prime} / L$-Asp/L-Phe-Gly on the $y$-axis; both at $20 \%$ NCE and 30 ms activation time. All results represent triplicate trials with standard deviations included 
aldohexoses was determined, the next step was to apply those same conditions to the eight ketohexoses. Only these fixed ligand combinations were pursued because they provided the best chiral discrimination among the aldohexose isomers. The thought process was that these two combinations should create significant enough energetic differences among all 24 isomers in order to accurately identify and determine absolute configuration for all the neutral hexose monosaccharides. Optimized mass spectrometric conditions found from the aldohexoses were applied for each of the eight ketohexose isomers with both fixed ligand combinations, $\mathrm{Mn}^{\mathrm{II}} / L$-Asp $/ L$-Phe - Gly and $\mathrm{Cu}^{\mathrm{II}} / L$-Ser/5'GMP. As previously stated, each isomer will yield a specific $\mathrm{R}_{\text {fixed }}$ range, for each fixed ligand combination used, based on averaged triplicate trials with standard deviation error bars.

Once again, if each of the 24 isomers has a unique and non-overlapping $\mathrm{R}_{\text {fixed }}$ range, simultaneous identification and absolute configuration determination is achieved. With data now collected for both the aldohexose and ketohexose isomers, at the same optimized conditions, a novel two-dimensional plot was created. Figure 6 shows the natural logarithm of $\mathrm{R}_{\text {fixed }}$ values for each isomer with the $\mathrm{Mn}^{\mathrm{II}} / L$-Asp $/ L$-Phe - Gly combination on the $\mathrm{y}$-axis and the natural logarithm of $\mathrm{R}_{\text {fixed }}$ values for each isomer with the $\mathrm{Cu}^{\mathrm{II}} / L$-Ser/5'GMP combination on the $\mathrm{x}$-axis. A natural $\log$-natural log plot was chosen because it was found easier to illustrate the data in small numerical ranges, compared with the initial raw data. (For each of the $\mathrm{R}_{\text {fixed }}$ values in raw data form, see the Supporting Information.) Figure 6 clearly shows all 24 hexose isomers discriminated from one another. Not only is each monosaccharide definitively identified, simultaneous absolute configuration determination is also achieved. This work represents the first such mass spectrometric discrimination, and simultaneous absolute configuration determination, of all 24 neutral hexose monosaccharide isomers.

\section{Comparison with Other Monosaccharide Analysis Techniques}

This method matches the low sample size requirements of HPAEC, GC/MS, and HPLC $(<5-50 \mu \mathrm{g})$ and reduces the amount as compared to NMR (milligram level) [9$11,16-29,52,53]$. However, this technique is able to determine absolute configuration, which HPAEC is unable to do, and there is no ambiguity in interpretation since only the relative intensities of two $\mathrm{MS}^{2}$ peaks are needed, compared with the convoluted chromatograms in GC/MS or HPLC and difficult spectra in NMR. Once the suitable fixed ligand combinations are found, each individual mass spectrometric run takes minutes, which provides a fast and accurate analysis. Lastly, there is no need for sample derivatization or for any home-built instrumentation, as a commercial mass spectrometer without any modifications was used for all the experiments.

\section{Conclusion}

Interestingly, in a study of the analysis of epimeric glycans with ion-mobility mass spectrometry, the presence of a single axial hydroxyl substituent on an aldohexose monosaccharide was hypothesized to lead to a lack of discrimination, as in the case of galactose (C4) versus mannose (C2) [6]. This single axial hydroxyl theory, however, does not hold in this fixed ligand method described. The three aldohexose monosaccharides with single axial hydroxyl substituents, allose (C3), galactose (C4), and mannose (C2), are all easily discriminated from one another based on their respective $\mathrm{R}_{\text {fixed }}$ ranges. Clearly, more studies that do not rely on data from only a small subset of carbohydrates are needed.

Herein the first analytical technique to identify any of the 24 neutral hexose monosaccharides and determine their absolute configuration (Figure 6) has been demonstrated. It can be foreseen that a similar strategy could be applicable for the definitive identification of other monosaccharides, such as the uronic acids, hexosamines, and 2-deoxysugars, which will ultimately allow the construction of mass-spectrometry-based protocols for de novo sequencing of glycans. This method can be readily adopted and employed for the identification of any unknown monosaccharide that is isolated from a biological or other source. Sample preparation requirements from one of these biological sources include for the monosaccharide to be free of any salts, which can be accomplished with dialysis followed by lyophilization $[54,55]$. In a biological sample, if the precursor ion is in low abundance, mass spectrometry conditions can be optimized to increase its abundance. Furthermore, as long as the precursor ion is in high enough abundance to be successfully trapped, there will be no issues with obtaining the desired pair of fragment ions and, thus, the fixed ligand kinetic method branching ratios. Work is ongoing to interface this mass-spectrometry-based monosaccharide identification method with a microfluidics oligosaccharide hydrolysis/ separation platform.

\section{Acknowledgments}

The authors acknowledge the Joan and Marvin Carmack Chair funds and the Indiana University Mass Spectrometry facility, especially Dr. Jonathan Karty and Dr. Jonathan Trinidad, the METACyt initiative, and the Eli Lilly Foundation for the funds to purchase the instrument used. They thank Professor David Clemmer and his labs for regular discussions.

\section{References}

1. Augusti, D.V., Carazza, F., Tao, W.A., Cooks, R.G.: Quantitative chiral analysis of sugars by electrospray ionization tandem mass spectrometry using modified amino acids as chiral reference compounds. Anal. Chem. 74, 3458-3462 (2002)

2. Gaucher, S.P., Leary, J.A.: Stereochemical differentiation of mannose, glucose, galactose, and talose using zinc(II) diethylenetriamine and ESIion trap mass spectrometry. Anal. Chem. 70, 3009-3014 (1998)

3. Xia, B., Zhou, Y., Xiao, J., Liu, Q., Gu, Y., Ding, L.: Use of electrospray ionization ion-trap tandem mass spectrometry and principal component 
analysis to directly distinguish monosaccharides. Rapid Commun. Mass Spectrom. 26, 1259-1264 (2012)

4. Mutenda, K.E., Matthiesen, R.: Analysis of carbohydrates by mass spectrometry. In: Matthiesen, R. (ed.) Methods in Molecular Biology, vol. 367, pp. 289-301. Humana Press, Totowa (2007)

5. Li, G., Huang, Z., Fu, C., Xu, P., Liu, Y., Zhao, Y.: L-valine assisted distinction between the stereo-isomers of D-hexoses by positive ion ESI tandem mass spectrometry. J. Mass Spectrom. 45, 643-650 (2010)

6. Both, P., Green, A.P., Gray, C.J., Sardzik, R., Voglmeir, J., Fontana, C., Austeri, M., Rejzek, M., Richardson, D., Field, R.A., Widmalm, G., Flitsch, S.L., Eyers, C.E.: Discrimination of epimeric glycans and glycopeptides using IM-MS and its potential for carbohydrate sequencing. Nat. Chem. 6 , 65-74 (2014)

7. Laine, R.: Invited commentary: a calculation of all possible oligosaccharide isomers both branched and linear yields $1.05 \times 10^{12}$ structures for a reducing hexasaccharide: the Isomer Barrier to development of singlemethod saccharide sequencing or synthesis systems. Glycobiology $\mathbf{6}$, 759-767 (1994)

8. National Research Council (US) Committee on Assessing the Importance and Impact of Glycomics and Glycosciences: Transforming Glycoscience: A Roadmap for The Future. National Academies Press, Washington, DC (2012)

9. Merkle, R.K., Poppe, I.: Carbohydrate composition analysis of glycoconjugates by gas-liquid chromatography/mass spectrometry. Methods Enzymol. 230, 1-15 (1990)

10. Ruiz-Matute, A.I., Hernandez-Hernandez, O., Rodriguez-Sanchez, S., Sanz, M.L., Martinez-Castro, I.: Derivatization of carbohydrates for GC and GC-MS analyses. J. Chromatogr., B 829, 1226-1240 (2010)

11. Bendiak, B., Fang, T.T.: End-group determination of oligosaccharides: a gas chromatography-mass spectrometry: mass spectrometry method for distinguishing between all D-aldohexoses and D-ketohexoses. Carbohydr. Res. 327, 463-481 (2000)

12. Arnaud, C.H.: Complex carbohydrates. Chem. Eng. News 92, 12-15 (2014)

13. Sassaki, G.L., de Souza, L.M.: Mass spectrometry strategies for structural analysis of carbohydrates and glycoconjugates. In: Coelho, A.V. (ed.) Tandem Mass Spectrometry-Molecular Characterization, pp. 81-115. InTech, Rijeka (2013)

14. Datta, D., Vaidehi, N., Floriano, W.B., Kim, K.S., Prasadarao, N.V., Goddard III, W.A.: Interaction of $E$. coli outer-membrane protein A with sugars on the receptors of the brain microvascular endothelial cells. Proteins 50, 213-221 (2003)

15. Aqvist, J., Mowbray, S.L.: Sugar recognition by a glucose/galactose receptor. Evaluation of binding energetics from molecular dynamics simulations. J. Biol. Chem. 17, 9978-9891 (1995)

16. Wu, L., Tao, W.A., Cooks, R.G.: Ligand and metal-ion effects in metal-ion clusters used for chiral analysis of alpha-hydroxy acids by the kinetic method. Anal. Bioanal. Chem. 373, 618-627 (2002)

17. Wu, L., Cooks, R.G.: Chiral analysis using the kinetic method with optimized fixed ligands: applications to some antibiotics. Anal. Chem. 75, 678684 (2003)

18. Wu, L., Tao, W.A., Cooks, R.G.: Kinetic method for the simultaneous chiral analysis of different amino acids in mixtures. J. Mass Spectrom. 38, 386-393 (2003)

19. Zhang, Z., Khan, N.M., Nunez, K.M., Chess, E.K., Szabo, C.M.: Complete monosaccharide analysis by high-performance anion-exchange chromatography with pulsed amperometric detection. Anal. Chem. 84, 4104-4110 (2012)

20. Fouquet, T., Charles, L.: Distinction and quantitation of sugar isomers in ternary mixtures using the kinetic method. J. Am. Soc. Mass Spectrom. 21, 60-67 (2010)

21. Tao, W.A., Cooks, R.G.: Peer reviewed: chiral analysis by MS. Anal. Chem. 75, 25-31 (2003)

22. Schug, K.A., Lindner, W.: Chiral molecular recognition for the detection and analysis of enantiomers by mass spectrometric methods. J. Sep. Sci. 28, 1932-1955 (2005)

23. Lindberg, B.: Methods for the structural elucidation of complex carbohydrates. In: Gregory, J.D., Jeanloz, R.W. (eds.) Glycoconjugate Research, vol. 1, pp. 17-34. Academic Press, New York (1979)

24. Wang, Y., Avula, B., Fu, X., Wang, M., Khan, I.A.: Simultaneous determination of the absolute configuration of 12 monosaccharide enantiomers from natural products in a single injection by a UPLC-UV/MS method. Planta Med. 78, 834-837 (2012)
25. Inoue, K., Kitahara, K., Aikawa, Y., Arai, S., Masuda-Hanada, T.: HPLC separation of all aldopentoses and aldohexoses on an anion-exchange stationary phase prepared from polystyrene-based copolymer and diamine:the effect of $\mathrm{NaOH}$ eluent concentration. Molecules 16, 5905-5915 (2011)

26. Ruhmann, B., Schmid, J., Sieber, V.: Fast carbohydrate analysis via liquid chromatography coupled with ultra violet and electrospray ionization ion trap detection in 96-well format. J. Chromatogr. A 1350, 44-50 (2014)

27. Stefansson, M., Novotny, M.: Electrophoretic resolution of monosaccharide enantiomers in borate-oligosaccharide complexation media. J. Am. Chem. Soc. 115, 11573-11580 (1993)

28. Lopes, J.F., Gaspar, E.M.S.M.: Simultaneous chromatographic separation of enantiomers, anomers, and structural isomers of some biologically relevant monosaccharides. J. Chromatogr. A 1188, 34-42 (2008)

29. Kuo, C., Liao, K., Liu, Y., Yang, W.: Bis-indole derivatives for polysaccharide compositional analysis and chiral resolution of D-, Lmonosaccharides by ligand exchange capillary electrophoresis using borate-cyclodextrin as a chiral selector. Molecules 16, 1682-1694 (2011)

30. Anumula, K.R.: Single tag for total carbohydrate analysis. Anal. Biochem. 457, 31-37 (2014)

31. Enders, J.R., McLean, J.A.: Chiral and structural analysis of biomolecules using mass spectrometry and ion mobility-mass spectrometry. Chirality 21, E253-E264 (2009)

32. Domalain, V., Huber-Roux, M., Lange, C.M., Baudoux, J., Rouden, J., Afonso, C.: Use of transition metals to improve the diastereomers differentiation by ion mobility and mass spectrometry. J. Mass Spectrom. 49, 423427 (2014)

33. Zhu, X., Sato, T.: The distinction of underivatized monosaccharides using electrospray ionization ion trap mass spectrometry. Rapid Commun. Mass Spectrom. 21, 191-198 (2007)

34. Salpin, J.Y., Tortajada, J.: Structural characterization of hexoses and pentoses using lead cationization. An electrospray ionization and tandem mass spectrometric study. J. Mass Spectrom. 37, 379-388 (2002)

35. Madhusudanan, K.P.: Tandem mass spectra of ammonium adducts of monosaccharides: differentiation of diastereomers. J. Mass Spectrom. 41, 1096-1104 (2006)

36. Young, B.L., Cooks, R.G.: Improvements in quantitative chiral determinations using the mass spectrometric kinetic method. Int. J. Mass Spectrom. 267, 199-204 (2007)

37. Yu, Y., Ko, K.S., Zea, C.J., Pohl, N.L.: Discovery of the chemical function of glycosidases: design, synthesis, and evaluation of mass-differentiated carbohydrate libraries. Org. Lett. 6, 2031-2033 (2004)

38. Zea, C.J., Pohl, N.L.: Kinetic and substrate binding analysis of phosphorylase $b$ via electrospray ionization mass spectrometry: a model for chemical proteomics of sugar phosphorylases. Anal. Biochem. 327, 107-113 (2004)

39. Karthikraj, R., Chitumalla, R.K., Bhanuprakash, K., Prabhakar, S., Vairamani, M.: Enantiomeric differentiation of $\beta$-amino alcohols under electrospray ionization mass spectrometric conditions. J. Mass Spectrom. 49, 108116 (2014)

40. Cooks, R.G., Ifa, D.R., Sharma, G., Tadjimukhamedov, F.K., Ouyang, Z.: Perspectives and retrospectives in mass spectrometry: one view. Eur. J. Mass Spectrom. 16, 283-300 (2010)

41. Wu, L., Cooks, R.G.: Chiral and isomeric analysis by electrospray ionization and sonic spray ionization using the fixed-ligand kinetic method. Eur. J. Mass Spectrom. 11, 231-242 (2005)

42. Lemr, K., Ranc, V., Fryack, P., Bednar, P., Sevcik, J.: Chiral analysis by mass spectrometry using the kinetic method in flow systems. J. Mass Spectrom. 41, 499-506 (2006)

43. Wu, L., Meurer, E.C., Young, B., Yang, P., Eberlin, M.N., Cooks, R.G.: Isomeric differentiation and quantification of $\alpha, \beta$-amino acid-containing tripeptides by the kinetic method: alkali metal-bound dimeric cluster ions. Int. J. Mass Spectrom. 231, 103-111 (2004)

44. Ming, L., Zhiqiang, L., Huanwen, C., Shuying, L., Qinhan, J.: Chiral quantification of D-, L-phenylglycine mixture using mass spectrometric kinetic method. J. Mass Spectrom. 40, 1072-1075 (2005)

45. Tao, W.A., Nikolaev, E.N., Cooks, R.G.: Copper (II)-assisted kinetic resolution of D, L-amino acids: a model system for chiral recognition in the gas phase. J. Am. Chem. Soc. 122, 10598-10609 (2000)

46. Kumari, S., Prabhakar, S., Sivaleela, T., Lakshmi, V.V.S., Vairamani, M.: Exploration of mononucleotides as fixed ligands towards chiral discrimination of hexose monosaccharides by the kinetic method. Eur. J. Mass Spectrom. 15, 35-43 (2009) 
47. Hyyrylainen, A.R.M., Pakarinen, J.M.H., Forro, E., Fulop, F., Vainiotalo, P.: Chiral differentiation of some cyclic $\beta$-amino acids by kinetic and fixed ligand methods. J. Mass Spectrom. 45, 198-204 (2010)

48. Lee, M., Kumar, A.P., Lee, Y.: Kinetic method for enantiomeric determination of thyroid hormone (D-L-thyroxine) using electrospray ionization tandem mass spectrometry (ESI-MS/MS). Int. J. Mass Spectrom. 272, 180$186(2008)$

49. Major, M., Fouquet, T., Charles, L.: Isomeric distinction of small oligosaccharides: a bottom-up approach using the kinetic method. J. Am. Soc. Mass Spectrom. 22, 1252-1259 (2011)

50. Gaspar, A., Lucio, M., Harir, M., Kopplin, P.: Targeted and non-targeted boron complex formation followed by electrospray Fourier transform ion cyclotron mass spectrometry: a novel approach for identifying boron esters with natural organic matter. Eur. J. Mass Spectrom. 17, 113-123 (2011)

51. Ricardo, A., Frye, F., Carrigan, M.A., Tipton, J.D., Powell, D.H., Benner, S.A.: 2-Hydroxymethylboronate as a reagent to detect carbohydrates: application to the analysis of the formose reaction. J. Org. Chem. 71, 9503-9505 (2006)

52. Konda, C., Londry, F.A., Bendiak, B., Xia, Y.: Assignment of the stereochemistry and anomeric configuration of sugars within oligosaccharides via overlapping disaccharide ladders using $\mathrm{MS}^{\mathrm{n}}$. J. Am. Soc. Mass Spectrom. 25, 1441-1450 (2014)

53. Rohrer, J.S., Basumallick, L., Hurum, D.: High-performance anion-exchange chromatography with pulsed amperometric detection for carbohydrate analysis of glycoproteins. Biochemistry (Mosc) 78, 697-709 (2013)

54. Packer, N.H., Lawson, M.A., Jardine, D.R., Redmond, J.W.: A general approach to desalting oligosaccharides released from glycoproteins. Glycoconj. J. 15, 737-747 (1998)

55. Sutherland, I.W.: Polysaccharides produced by Cystobacter, Archangium, Sorangium, and Stigmatella species. J. Gen. Microbiol. 111, 211-216 (1979) 